\title{
EETA: An Energy Efficient Transmission Alignment for Wireless Sensor Network Applications
}

\author{
Sangsoon Lim ${ }^{1}$, Hayoung $\mathrm{Oh}^{2}$ \\ ${ }^{1}$ Software R\&D Center, Samsung Electronics, Seoul, South Korea \\ ${ }^{2}$ School of Electronic and Engineering, SoongSil University, Seoul, South Korea
}

\begin{tabular}{l} 
Article Info \\
\hline Article history: \\
Received Apr 10, 2016 \\
Revised Jun 1, 2016 \\
Accepted Jun 17, 2016 \\
\hline
\end{tabular}

Keyword:

Application traffic Energy efficiency

MAC protocol

Wireless sensor networks

\begin{abstract}
Energy conserving MAC protocols performing adaptive duty-cycling mechanism have been widely studied to improve the energy efficiency in Wireless Sensor Networks (WSNs). In particular, several asynchronous low power listening (LPL) MAC protocols such as B-MAC, X-MAC and ContikiMAC transmit a long preamble or consecutive data packets for an efficient rendezvous between senders and receivers. However, the rendezvous results in the challenging problem of unnecessary channel utilization since the senders occupy a large portion of the medium. Furthermore, when a traffic generation time overlaps with other neighbouring nodes, they frequently encounter spatially-correlated contention incurring excessive channel contention. In this paper, we propose a novel traffic distribution scheme called an Energy Efficient Transmission Alignment (EETA), that shifts a traffic generation time of the application layer. By using a MAC layer feedback including contention information, the cross-layer framework determines whether the node delays its transmission or not. EETA is robust from the heavy contending environment due to its traffic distribution feature. We evaluate the performance of EETA through diverse experiments on the TelosB platform. The results show that EETA improves the overall energy efficiency by up to $35 \%$, and reduces the latency by up to $48 \%$ compared to the existing scheme.
\end{abstract}

Copyright () 2016 Institute of Advanced Engineering and Science. All rights reserved.

\section{Corresponding Author:}

Hayoung Oh,

School of Electronic and Engineering,

SoongSil University,

Seoul, South Korea.

Email: hyoh79@gmail.com

\section{INTRODUCTION}

Wireless Sensor Network (WSN) is an emerging technology that supports low-power and reliable wireless communication of various real-life applications [1]. It has a great potential to enable novel smart services in the Internet of Things (IoT) environment [2]. Since WSNs are made up of tiny battery-powered sensor nodes, one of the most critical challenges is energy consumption problem [3].

Several clever schemes have been proposed [4]-[8] in MAC layer to deal with the problem. The schemes can be categorized into two types according to duty-cycling mechanisms: synchronous and asynchronous approaches. In the synchronous approaches [4],[5], all nodes deployed in the same network domain simultaneously turn the RF transceivers on and off to synchronize their transmission schedules while performing low power operation. Although it preserves more energy in a specific environment, clock synchronization limits the performance improvements in the wireless environment. In the asynchronous approaches [6]-[8], a receiver checks the on-going transmissions every wake-up interval. It makes use of Clear Channel Assessment (CCA) mechanism to identify ZigBee data packets. If the intended receiver senses a busy channel, it keeps the wake-up state for the potential packet. A sender continuously transmits a long 
preamble or data packets until receiving an acknowledge packet or reaching the end of the wake-up interval. The asynchronous schemes have been implemented on TinyOS [9] and Contiki OS [10], and widely used. These protocols enable a ZigBee transceiver to operate at low-duty cycle without any time synchronization and provide simplicity and effectiveness.

However, the asynchronous Low Power Listening (LPL) MAC protocols such as B-MAC [6], XMAC [7] and ContikiMAC [8] occupy a large portion of the medium during the rendezvous between senders and receivers. It significantly affects the unnecessary channel contentions in most of WSN applications. Many WSN applications report their information such as event data, monitoring data and control data by periods [11]. In addition, a central entity, called sink node, gathers those information from all nodes in the same domain network. Since the transmissions have a spatial correlation, excessive data transmissions occur at the same time in the same interference region. In this case, the proliferation of data packets causes additional channel contentions and results in severe packet collisions among neighbourhood nodes. It aggravates the energy efficiency, end-to-end delay and packet reception ratio in the real WSN deployment.

In this paper, we propose a novel traffic alignment scheme, called EETA, to alleviate the spatiallycorrelated contention problem in WSNs. EETA analyses a backoff pattern in MAC layer and provides useful feedbacks to the application layer. It measures a busy period while sensing the channel status to transmit data packets. Based on the measured busy period from transmissions of other neighbourhood nodes, the application layer shifts its initial transmission point of the periodic traffic. EETA aims at slightly avoiding overlapping of the channel usage pattern between nodes located in the same interference region. Thus, it considerably reduces channel contention delay and unnecessary energy drain in the channel contention phase. We implemented EETA on the TelosB sensor node [12] to verify the feasibility of the traffic alignment scheme. Furthermore, we evaluate the performance of our scheme in terms of the energy efficiency, end-toend delay and packet reception ratio. The results show that our proposed scheme reduces the energy consumption by up to $35 \%$, end-to-end delay by up to $48 \%$. The performance improvement increases as the number of neighbourhood nodes increase.

The rest of this paper proceeds as follows. Section 2 introduces the related work. We then describe the application traffic alignment scheme in Section 3. Section 4 evaluates the proposed scheme. Finally, Section 5 concludes the paper.

\section{RELATED WORK}

\subsection{Wireless Sensor Network Applications}

WSNs are applied in diverse areas such as smart building, smart home, smart healthcare, and so on [13]. Smart buildings try to reduce energy consumption by appropriate humidity, ventilation, air conditioning (HVAC) control and measure room occupancy, temperature, and various features of the facilities. Smart home application intelligently checks environmental status or human activities and controls home environments with various actuators. Smart healthcare focuses on monitoring people's daily life and providing medical alarm under certain medical conditions. In these scenarios, multiple sensor nodes are located in the same geographic area for the purpose of reliability. In addition, when an interesting event happens, multiple nodes generate a periodic traffic to convey their measured data or control messages. It thus leads to spatially-correlated channel contention. In this case, a lot of sensor nodes concurrently transmit data packets in response to the same event. Especially, these phenomena degrade channel quality, thereby aggravating the overall performance of WSN applications. Our proposed scheme can avoid the spatiallycorrelated channel contention via an efficient application traffic alignment.

\subsection{Sensor MAC Protocols}

S-MAC [4] is one of the most well-known energy conserving MAC protocols in WSNs. Each node employing S-MAC simultaneously turns its RF transceiver off to conserve the energy consumption during idle listening period. Senders and receivers periodically share the synchronization information to communicate each other during active period. In [5], T-MAC dynamically adjusts active and sleep periods by considering communication patterns and hardware limitation. Although these solutions address the problem of energy waste due to idle listening, the networks severely suffer from synchronization overheads to maintain wake-up schedules.

Another approach to preserve energy wastes is asynchronous mechanism proposed in B-MAC [6]. B-MAC makes use of a long preamble concept instead of static time synchronization. Each receiver periodically wakes up every wake-up interval. A sender transmits a long preamble during the entire wake-up interval when the node has a packet to send. Therefore, they can success their transmissions within the wakeup interval. This scheme does not need to exchange synchronization information. To additionally reduce the 
heavy burdens of the long preamble, X-MAC [7] consecutively transmits short preambles until the intended receiver receives the short preamble and sends an early-ack. In particular, ContikiMAC [8], widely used in real WSN testbeds, combines the advantages of various asynchronous approaches such as B-MAC, X-MAC, and BoX-MAC [14]. However, these solutions incur excessive channel contention due to a long preamble or many successive data packets of senders. Our proposed scheme deals with the channel contention problem of the asynchronous LPL MAC protocols by providing MAC layer contention feedback to the application layer.

\section{ENERGY EFFICIENT TRAFFIC ALIGNMENT}

In this section, we first point out a channel contention problem of current WSN MAC protocols and then propose energy efficient traffic alignment (EETA) framework and algorithm.

\subsection{Channel Contention Overhead of energy conserving MAC protocols}

In a low duty cycle environment of WSNs, the energy consumption of the reception operation suffering from idle listening and overhearing is more severe problem than the transmission operation [12]. Therefore, most of the existing asynchronous low power MAC protocols in WSNs adopt a large preamble or consecutive data transmissions to rendezvous between a sender and a receiver. However, this approach causes heavy channel contention overhead. Figure 1 illustrates the channel contention overhead of the asynchronous low power MAC protocol employed in ContikiOS. A sender needs to transmit consecutive data packets during the entire wake-up interval. If a traffic arrival of the application layer occurs at the same time among neighbourhood nodes, they severely suffer from excessive channel contention due to consecutive data packets. During the channel contention, every node must keep idle listening to check a channel status. It drastically degrades the overall performance of the network. To overcome the inefficiency, we devise EETA to evenly distribute application traffics among nodes. We will explain the core framework of EETA in the next sub-section.

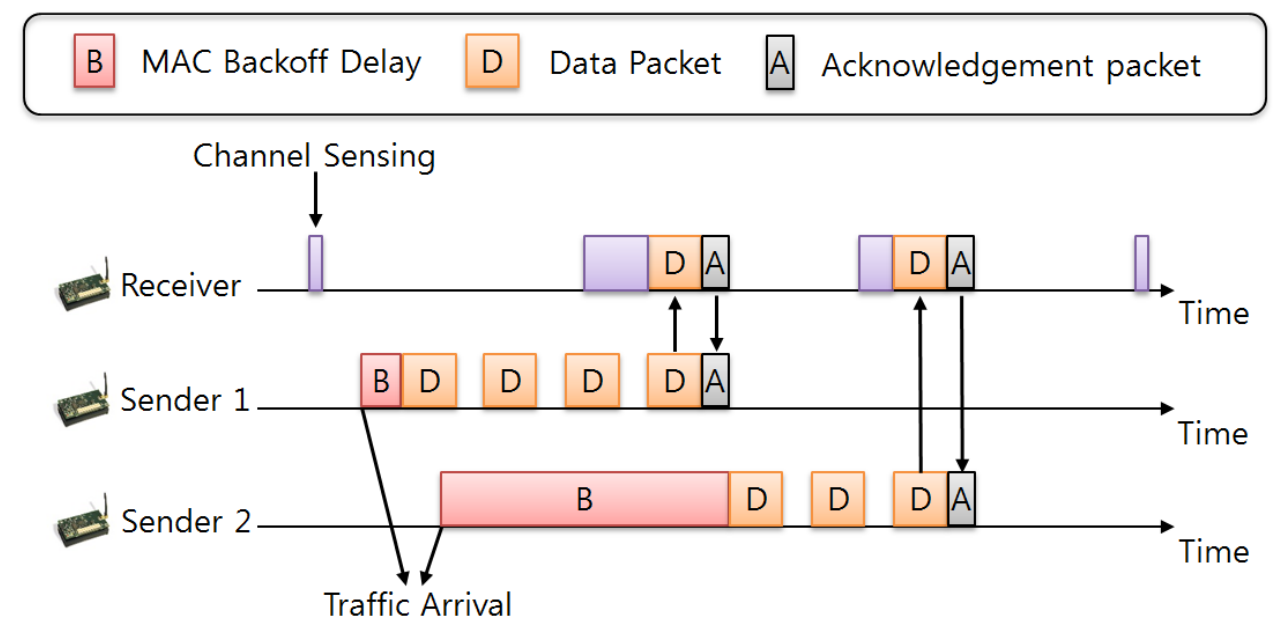

Figure 1. Channel contention overhead of asynchronous low power MAC protocol

\subsection{Cross-layer Framework of EETA}

EETA is designed for an efficient transmission distribution to reduce the energy consumption of each sensor node. Figure 2 shows the framework of EETA. In case of MAC layer, the contention monitor module checks a backoff delay after the node generates its data packet. The backoff delay statistics are then processed by a contention analysing module that decides whether it is normal contention or not in the contention analyse process. The decision result is then processed by a cross-layer feedback module to notify it to the application layer. In case of application layer, the traffic alignment module shifts its transmission start point of every periodic data packet based on the mac layer feedbacks. A key advantage of traffic shifting is that it can minimize the channel contention cost, thereby improving channel utilization and reducing idle listening of the node. The feedback information includes the number of the consecutive failures of Clear Channel Assessment (CCA), which performs energy-based channel sensing to check whether a channel is busy or idle. When other sensor nodes occupy a large portion of the medium due to their low power operation, the CCA may successively fail until the other pairs of nodes are done with their transmissions. In 
addition, a wake-up interval of MAC protocol is another parameter to schedule application traffics. To support these features, we design a cross-layer software framework between MAC and application layers. When the MAC layer analyses contention patterns, the framework immediately adjusts the packet transmission schedule.

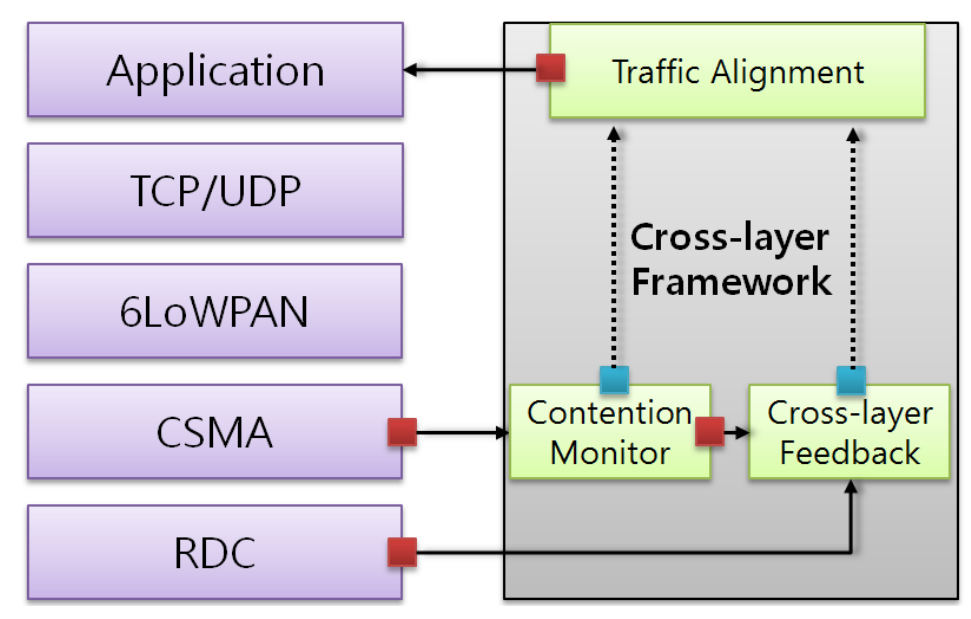

Figure 2. Cross-layer framework of energy efficient traffic alignment

\subsection{Traffic Alignment Algorithm}

Current long preamble over low power operation constitutes a major part of excessive channel contention in WSNs. Therefore, it is necessary that each node avoids concurrent transmissions of a periodic data packet that overlaps with the activities of other nodes. According to channel usage patterns of neighbourhood nodes, there is a stable pattern for the event-driven WSN application. Each node measures its backoff delay $\mathrm{T}_{\mathrm{D}}$ per one application packet in a Carrier Sensing Multiple Access (CSMA) sub-layer performing channel contention process, once the original packet is generated by the application layer. In addition, it checks a wake-up interval $\mathrm{T}_{\mathrm{w}}$ from a Radio Duty Cycle (RDC) module to calculate the average transmission delay of low power operation. The algorithm for traffic alignment is given in Algorithm 1. In every run, the inputs to the algorithm are $T_{D}$ and $T_{w}$ and the output is traffic shifting offset, $T_{S}$. First, the sender computes the current MAC layer backoff delay and adds it to $T_{D}$. If $T_{D}$ is larger than the average wake-up interval and the node suffers from consecutive backoff, the node increases the number of backoff trial, $\mathrm{N}_{\mathrm{B}}$. And then, the node computes the culumative MAC layer backoff delay to calculate the traffic shifting offset when the $\mathrm{N}_{\mathrm{B}}$ exceeds a specific threshold . Eventually, although there exist several nodes attempting to transtmit at the same time in the interfering region, they slightly shifts their traffic generation times and can avoid unnecessary contention time causing energy wastes.

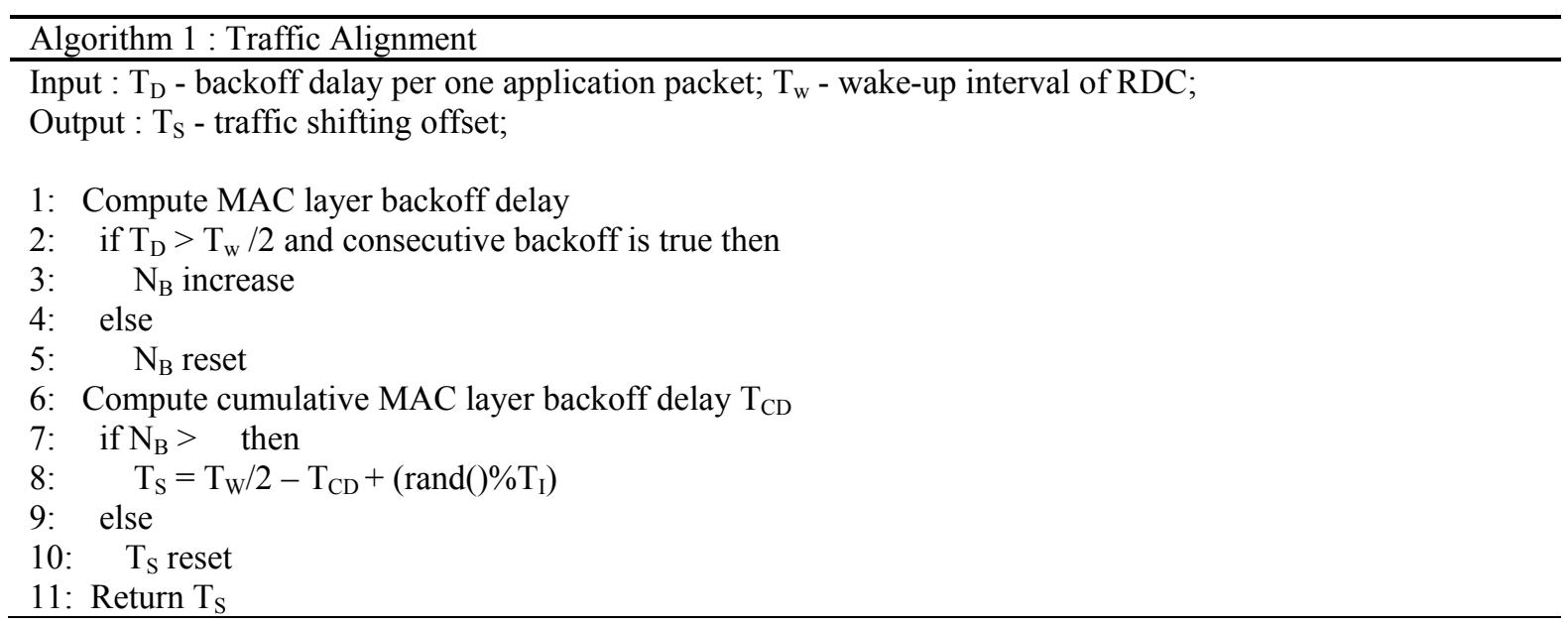




\section{PERFORMANCE EVALUATION}

\subsection{Experimental Setup}

We have implemented on TelosB sensor nodes [12] running Contiki OS 2.7. TelosB is made up of a MSP430 Micro Control Unit (MCU) and a CC2420 radio chipset. The CC2420 chipset is an 802.15.4 compliant RF transceiver operating in the $2.4 \mathrm{GHz}$ ISM band and supports a data rate of $250 \mathrm{kbps}$. We make use of the Contiki MAC module developed by dunkels [10] and add MAC layer feedback mechanism. To the sender side, we embedded traffic alignment function for traffic distribution. We set one receiver and nine senders to evaluate our proposed scheme. To explore the effect of the wake-up interval duration, we vary the wake-up interval from $62.5 \mathrm{~ms}$ to $250 \mathrm{~ms}$ in the same distance. In our experiment, we analyse the energy consumption by comparing the sojourn time at active and sleep state. We also measure end-to-end delay of application traffics to verify the feasibility of EETA.

\subsection{Energy Consumption}

Figure 3 shows the experimental result for the average energy consumption per unit time of paired sender and receiver as a function of the wake-up intervals. In both basic LPL MAC and LPL MAC with EETA, the receiver saves more energy as the wake-up interval increases due to a large portion of sleep duration. When a node performs duty-cycling mechanism with long wake-up interval, the node keeps sleep state avoiding unnecessary energy drain during idle state, thereby the scheme can improve the energy efficiency. When several senders transmit their packet at the same time, only one sender gets the medium during the channel contention phase. Thus, other nodes wait for the next opportunity while performing additional channel backoff. In that case, the basic LPL MAC severely suffers from channel contention overhead, thereby it leads to increase idle listening duration for an additional channel backoff. However, our proposed scheme slightly shifts its traffic generation time to mitigate spatially-correlated contention among neighbouring nodes. After a node senses an excessive channel contention, it adaptively adjusts its periodic traffic pattern. As a result, LPL MAC with EETA conserves the average energy consumption compared to the existing LPL MAC by up to $35 \%$ due to the effect of traffic alignment.

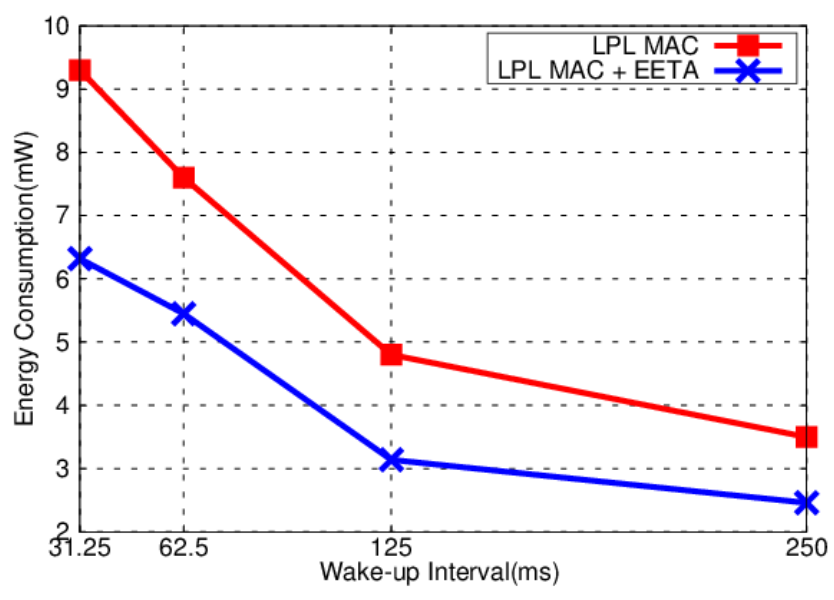

Figure 3. Average energy consumption with respect to the wake-up intervals

\subsection{End-To-End Delay}

Figure 4 describes the experimental result for the latency as a function of wake-up intervals. In LPL MAC, both a sender and a receiver do not know the exact wake-up point of each other. It incurs rendezvous delay between the sender and the intended receiver. Therefore, as the wake-up interval increases, the average delay also increases. When the nodes perform EETA to avoid concurrent transmissions, they are considerably able to reduce the latency compared to the basic LPL MAC by up to $48 \%$. Since the application traffics are evenly distributed, simultaneous transmission trials can be reduced. It indicates that a node can have more opportunity to grab the medium with less channel contention and minimize idle listening duration for unnecessary channel contentions. In case of LPL MAC with EETA, the delay variation is smaller than the LPL MAC. Since EETA adaptively controls the contention environment, it effectively reduces the random property of an additional channel backoff and unnecessary waiting time for a transmission. 


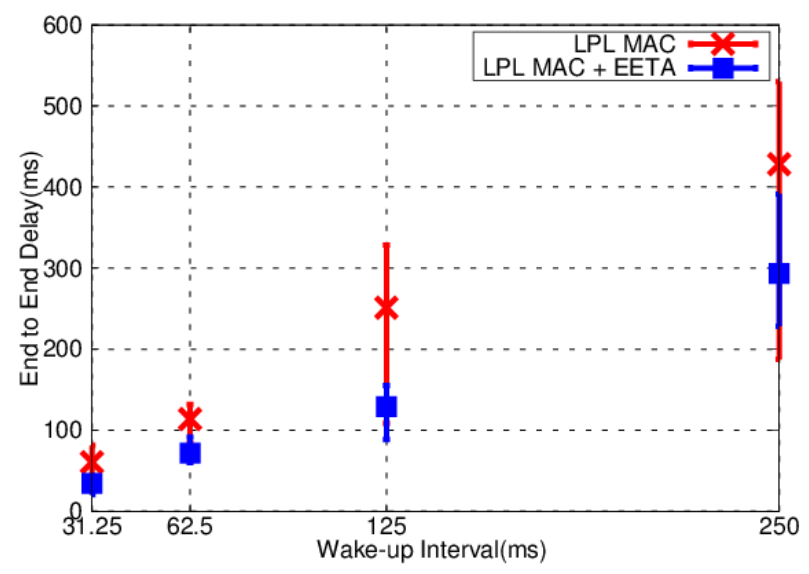

Figure 4. Latency with respect to the wake-up intervals

\subsection{Packet Reception Ratio}

Figure 5 demonstrates the packet reception rate as a function of concurrent transmission nodes. In WSNs, periodic data packet is one of the main features for most of the applications. For example, several sensor nodes periodically report diverse information to the central coordinator, called sink node. In addition, some nodes located in the same coverage transmit periodic information after sensing a special event. To verify our proposed scheme, all neighbouring nodes simultaneously generate periodic traffic every 5 seconds. As the number of concurrent packet transmissions increases, the packet reception rate of LPL MAC is significantly degraded. The reason is that 802.15 .4 has its own limitation of channel contention. If a node fails to grab the medium during the limitation, the transmission trial also fails. As a result, it aggravates packet reception rate of LPL MAC. However, our scheme does not exceed the channel contention limitation of 802.15.4. When the channel contention duration exceeds a specific threshold, it intelligently avoids the channel contention. Therefore, LPL MAC with EETA outperforms the vanilla LPL MAC. This shows that EETA is more practical solution in a real environment that consists of many sensor nodes. Our proposed scheme is well-matched with the heterogeneous wireless network environment due to the adaptation algorithm.

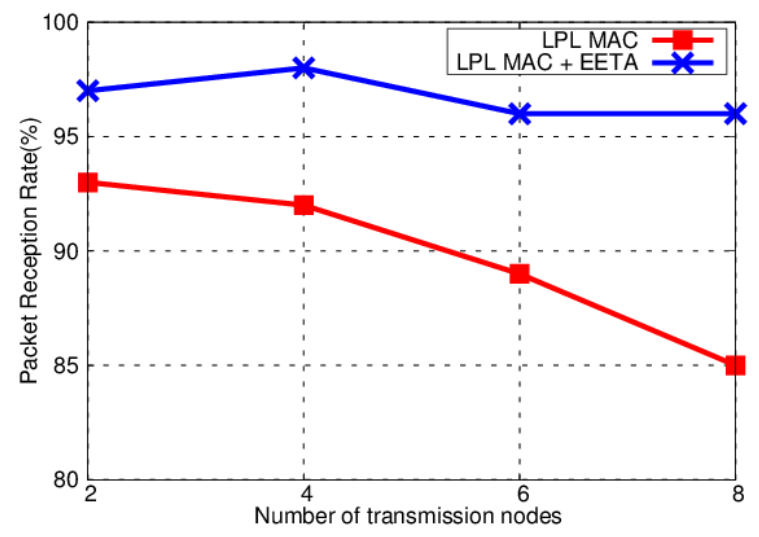

Figure 5. Packet reception rate with respect to number of concurrent transmission nodes

\section{CONCLUSION}

In WSNs, diverse energy conserving protocols based on duty-cycling mechanism have been widely used in MAC layer because of its efficiency. However, the heavy burdens of low power operations in MAC layer severely degrade the overall performance of low power protocols. In particular, when a transmission occurs at the same time, it incurs unnecessary energy waste due to the excessive channel contention of periodic data transmissions. We proposed a novel traffic alignment scheme, named EETA, which evenly 
distributes application traffics. We designed cross-layer framework to fully utilize MAC layer feedback and shift application traffics. To verify its feasibility, we implemented our proposed scheme on TelosB sensor nodes. Our experimental results showed that our scheme improves energy efficiency and end-to-end delay compared to the existing scheme.

\section{ACKNOWLEDGEMENTS}

This research was supported by Basic Science Research Program through the National Research Foundation of Korea (NRF) funded by the Ministry of Science, ICT \& Future Planning (NRF2014R1A1A1003562).

\section{REFERENCES}

[1] R. Khadim, et al., "Performance Study of IEEE 802.15. 4 under OPNET Modeler for Wireless Sensor Networks," TELKOMNIKA Indonesian Journal of Electrical Engineering, vol/issue: 16(1), pp. 98-105, 2015.

[2] N. Dinh and S. Lim, "Analysis of IEEE 802.15.4 Beacon-Enabled MAC Protocol," IJECE, vol/issue: 6(3), 2016.

[3] Akyildiz, et al., "A survey on sensor networks," IEEE Communications Magazine, vol/issue: 40(8), pp. 102-114, 2002.

[4] W. Ye, et al., "An energy efficient mac protocol for wireless sensor networks," in 21st International Annual Joint Conference of the IEEE Computer and Communications Societies, 2002.

[5] T. van Dam and K. Langendoen, "An adaptive energy-effcient MAC protocol for wireless sensor networks," in Proc. of the First ACM Conference on Embedded Networked Sensor Systems, 2003.

[6] J. Polastre, et al., "Versatile low power media access for wireless sensor networks," in Proc. of the 2nd ACM SenSys Conference, 2004.

[7] M. Buettner, et al., "X-MAC: A Short Preamble MAC Protocol for Duty-Cycled Wireless Sensor Networks," in Proc. of the 4th ACM SenSys Conference, 2006.

[8] A. Dunkels, "The contikimac radio duty cycling protocol," Technical Report 5128, Swedish Institute of Computer Science, 2011.

[9] TinyOS, http://www.tinyos.net.

[10] ContikiOS, http://www.contikios.org.

[11] U. Prathap, et al., "Wireless sensor networks applications and routing protocols: survey and research challenges," Cloud and Services Computing (ISCOS), 2012 International Symposium on. IEEE, 2012.

[12] TelosB, http://www.memsic.com/userfiles/files/Datasheets/WSN/telosb_datasheet.pdf

[13] A. Al-Fuqaha, et al., "Internet of things: A survey on enabling technologies, protocols, and applications," Communications Surveys \& Tutorials, IEEE, vol/issue: 17(4), pp. 2347-2376, 2015.

[14] D. Moss and P. Levis, "BoX-MACs: Exploiting physical and link layer boundaries in low-power networking," Computer Systems Laboratory Stanford University, pp. 116-119, 2008.

\section{BIOGRAPHIES OF AUTHORS}

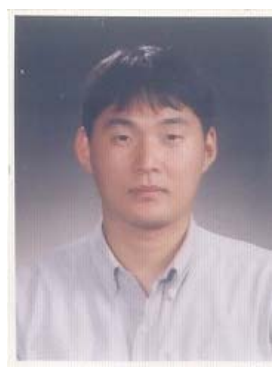

Sangsoon Lim received $\mathrm{Ph}$. D. degree in the School of Computer Science and Engineering from Seoul National University in 2013. Since October 2013, he works as a senior engineer at Software R\&D Center, Samsung Electronics. His current research interests are in the area of wireless networks including wireless LAN, wireless sensor Networks, cognitive radio networks.

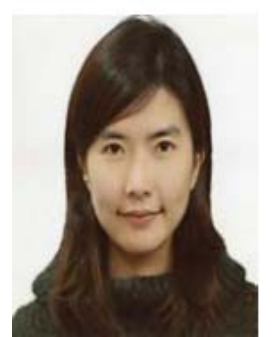

Hayoung Oh received the B.S. degree in Computer Science from Duksung Womans University and the M.S. degree in the School of Computer Science and Engineering from Ewha Womans University in 2002 and 2006 respectively. And she received the Ph.D. degree in Computer Science from Seoul National University in 2013. From 2002 to 2004, she joined Shinhan Financial Group as a developer in applied research. In 2010, she was with U.C. Berkeley as a researcher. Since 2013, she has been with Soongsil University as a professor in the School of Electronic Engineering. Her research interests include social and computer networks, and security. 